\section{Secrets of plants: Endophytes}

\author{
Asiya Nazir, Habeeb Abdul Rahman \\ Department of Applied Science and \\ Mathematics, College of Arts and \\ Sciences, Abu Dhabi University, Abu \\ Dhabi, United Arab Emirates
}

\begin{abstract}
Endophytic fungi are an important component that colonizes in healthy tissues of living plants and can be readily isolated from any microbial or plant growth medium. They act as reservoirs of novel bioactive secondary metabolites, such as alkaloids, phenolic acids, quinones, steroids, saponins, tannins, and terpenoids that serve as a potential candidate for antimicrobial, anti-insect, anticancer and many more properties. Their huge diversity and particular habituation, they can provide a good area for research in the field of making new medicines and novel drug-like molecules. Because of the impact of endophytes on host plant by enhancing their growth or increasing their fitness, also making them tolerant to abiotic and biotic stresses and holding the secondary metabolites, endophytes are gaining attention as a subject for research. This review aims to comprehend the contribution and uses of endophytes and relationships between endophytic fungi and their host medicinal plants.
\end{abstract}

\section{Introduction}

Endophytes are microorganisms that reside within study plant tissues intercellularly and/or intracellularly but usually remain asymptomatic and does not show any noticeable damage to the host. Endophytic fungi inhabit plant tissues without destroying or producing substances that cause an infection in the host cell. They exhibit complex interactions with their hosts, which involves mutualism and antagonism. ${ }^{1,2}$ Plants strictly limit the growth of endophytes, and these endophytes use many mechanisms to gradually adapt to their living environments. ${ }^{3}$ Unlike mycorrhizal fungi that colonize plant roots and grow into the rhizosphere, endophytes reside entirely within plant tissues and may grow within roots, stems and/or leaves, emerging to sporulate at plant or host $\square$ tissue senescence. ${ }^{1,4}$ Symbioses have been responsible for the origin of major ecological and evolutionary transitions in the history of life. Plants are hosts to complex communities of endophytes that colonize the interior of both below- and above-ground tissues. The endophytic fungus grows asymptomatically in aerial plant tissues and is vertically transmitted from the plant to its offspring via seeds and tillers. Endophytes are thought to interact mutualistically with their host plants mainly by increasing host resistance to herbivores ${ }^{1}$ and have been termed acquired plant defenses. ${ }^{5}$ In return, plants provide spatial structure and protection from desiccation, nutrients, and photosynthate and, in the case of vertical-transmission, dissemination to the next generation of hosts. Endophytic fungi represent an important and quantifiable component of fungal biodiversity in plants that impinge on plant community diversity and structure.

They produce a wide range of compounds useful for plants for their growth, protection to environmental conditions, and sustainability, in favor of a good dwelling place within the hosts. Due to its ability of producing bioactive secondary metabolites similar or same compounds as their host plant, promoting growth, good yield, inducing host plants to tolerate both biotic and abiotic stresses and disease resistance, endophytes is currently attracting a lot of research interest and therefore, can be used as a potential source of novel natural products for food, industrial, medicinal and agricultural industries.

\section{Classification of endophytes}

There are mainly two classes in which endophytic fungi are classified as Class I Clavicipitaceae and Class II Nonclavicipitaceous. The Class 1 endophytes are the clavicipitaceous endophytes associated with warm- and cool-season grasses. They are host specific, mainly in the grass family poaceae and rarely in cyperaceae, and are often vertically transmitted through seeds. Transmission of Class 1 endophytes is primarily vertical, with maternal plants passing fungi on to offspring via seed infections. ${ }^{6}$ Class 1 endophytes frequently increase plant biomass, confer drought tolerance, and produce chemicals that are toxic to animals and decrease herbivory. ${ }^{5}$ However, the benefits conferred by these fungi appear to depend on the host species, host genotype and environmental conditions. ${ }^{7,8}$

Compared with Class 1 endophytes, Class 2 endophytes are highly diverse and comprised of species from pezizomycotina (ascomycota) to agaricomycotina and pucciniomycotina (basidiomycota). ${ }^{9}$ They colonize roots, stems, leaves, or the whole plant. They can be vertically or horizontally transmitted. However, NC-endophytes represent three distinct functional classes class 2, 3 and 4, based on host colonization and
Correspondence: Asiya Nazir, Department of Applied Science and Mathematics, College of Arts and Sciences, Abu Dhabi University, Abu Dhabi, United Arab Emirates.

Tel.: +97125015447.

E-mail: asiya.nazir@adu.ac.ae

Key words: Endophytic fungi; bioactive compounds; secondary metabolite.

Contributions: the authors contributed equally.

Conflict of interest: the authors declare no potential conflict of interest.

Received for publication: 5 August 2018.

Revision received: 5 September 2018.

Accepted for publication: 6 September 2018.

This work is licensed under a Creative Commons Attribution-NonCommercial 4.0 International License (CC BY-NC 4.0).

(C) Copyright A. Nazir and H.A. Rahman, 2018 Licensee PAGEPress srl, Italy

International Journal of Plant Biology 2018; 9:7810 doi:10.4081/pb.2018.7810

transmission, in plant biodiversity and fitness benefits conferred to hosts while the $\mathrm{C}$ group has just one class. Class 2 endophytes have been the most extensively researched and has been shown to enhance fitness benefits of their plant host as a result of habitatspecific stresses such as $\mathrm{pH}$, temperature, and salinity. Class 3 endophytes are restricted to growth in belowground plant tissues and form in localized areas of plant tissue. Class 4 endophytes are also restricted to plant tissues below ground but can colonize much more of the plant tissue. ${ }^{10} \mathrm{NC}$-endophytes have been recovered from every major lineage of land plants, and from all terrestrial ecosystems, including both agroecosystems and biomes ranging from the tropics to the tundra. ${ }^{11}$

The scale of their diversity, their ecological roles, the insights they provide into the evolution of various ecological modes in fungi, their potential applications, and the ability of many fungi to switch between endophytic and free $\square$ living lifestyles ${ }^{12,13}$ are becoming more apparent, engendering growing enthusiasm from mycologists, ecologists, physiologists and applied scientists.

\section{Isolation and identification}

Endophytic fungi are any fungi that grow all or part of their life cycles symptomlessly in the intercellular spaces of living and apparently healthy host plant tis- 
sues, while the inhabited host tissues remain intact and functional. The diversity and numbers of fungal endophytes vary considerably and their detection depends on biotic, abiotic and experimental factors. Detection and recovery of endophytic fungi have relied heavily on dissection of plant organs into small fragments followed by their surface sterilization and subsequent plating of fragments onto a nutrient-rich agar medium. ${ }^{4,14}$

Traditionally, endophytic fungi inside plant tissues can be recognized by two basic techniques, i.e. direct observation and cultivation-dependent methods. In the direct observation method, endophytic fungal structures within living plant tissues are directly examined under a light and electron microscope, which can show all endophytic mycobiota within the plant tissue. ${ }^{15}$ In contrast, cultivation-dependent techniques have been routinely employed in endophyte diversity studies. ${ }^{1}$ It is important to isolate endophytic fungi for further detailed studies into their characterization, population dynamics, species diversity, or as inoculate to improve plant growth and health, or screening for novel biologically active secondary metabolites (Figure 1).

Endophytes can also be isolated by culturing from ground tissue extract ${ }^{16}$ or by direct culturing of plant tissues ${ }^{17}$ on media suitable for bacteria or fungi or actinomycetes. Conventionally, identification of endophytes is based on morphological characteristics for bacteria, fungi, and actinomycetes and with the help of biochemical tests for bacteria and actinomycetes. With the development of molecular biology, ribosomal DNA Internal Transcribed Spacer (ITS) sequence analysis is widely used for the identification of microorganisms. Ribosomal DNA (rDNA) ITS was proved to be a valuable source of evidence to resolve phylogenetic relationships at lower levels, such as among genera or species. ${ }^{18}$

Microorganisms are important mediators of plant-herbivore interactions. The role of endophytic microorganisms in plant associations has been extensively discussed. Endophytes are considered plant mutualists. They receive nutrition and protection from the host plant while the host plant may benefit from enhanced competitive abilities and increased resistance to herbivores, pathogens, and various abiotic stresses. ${ }^{7}$ As endophytes colonize ecological niches similar to those colonized by phytopathogens, interactions between these two groups are possible, and in fact, many studies have shown that endophytic microorganisms isolated from surface disinfected plant tissues exhibit potential as biocontrol agents against phytopathogens and insects. ${ }^{19}$ Reports of molecular studies on endophytic bacterial diversity have revealed a large richness of species. Endophytes promote plant growth and yield, suppress pathogens, may help to remove contaminants, solubilize phosphate, or contribute assimilable nitrogen to plants. ${ }^{20}$ The endophyte-host relationship is believed to be complex and probably varies from host to host and microorganism to the microorganism. ${ }^{21}$ Many experiments have been conducted to compare how endophyte-infected plants and non-infected plants behave in relation to environmental stress, and attack by insect and animal predators. ${ }^{22}$ Therefore, search for interesting biological activities within the natural biodiversity has been the basis for the development of various applications in biotechnology, agriculture, production of pharmaceuticals compounds and other fields.

\section{Application of endophytes}

Medicinal plants and their endophytes are important resources for the discovery of natural products. Since endophytes residing inside healthy plant tissues without any discernible infectious symptom and exhibit mutualism and antagonism with the host, they could be a potential source of novel natural products for medicinal, agricultural, and industrial uses. Endophytes provide a wide variety of structurally unique bioactive natural products, such as alkaloids, benzopyranones, chinones, flavonoids, phenolic acids, quinones, steroids, terpenoids, tetralones, xanthones, and others. ${ }^{23}$

\section{Antioxidants and phenolic con- tent of endophytes}

Antioxidants are thought to be highly effective in the management of ROS-mediated tissue impairments. Many antioxidant compounds possess anti-inflammatory, anti-atherosclerotic, antitumor, antimutagenic, anticarcinogenic, antibacterial, and antiviral activities to a greater or lesser extent. Afra et al., in 2015, revealed that endophytic fungus Aspergillus sp. from Trigonella foenum-graecum seeds demonstrated the highest both total phenolic content in term of gallic acid equivalent and antioxidant activity for free radical scavenging assay. ${ }^{24}$ The antioxidant activity and total phenolic content (TPC) of ethyl acetate extracts of endophytic fungi isolated from Eugenia jambolana were studied by Manila et al., in 2014. ${ }^{25}$ The study showed that $36 \%$ endophytic extracts of Chaetomium sp., Aspergillus sp., Aspergillus peyronelii and Aspergillus Niger was having high phenolic content exhibited potent antioxidant activity. The

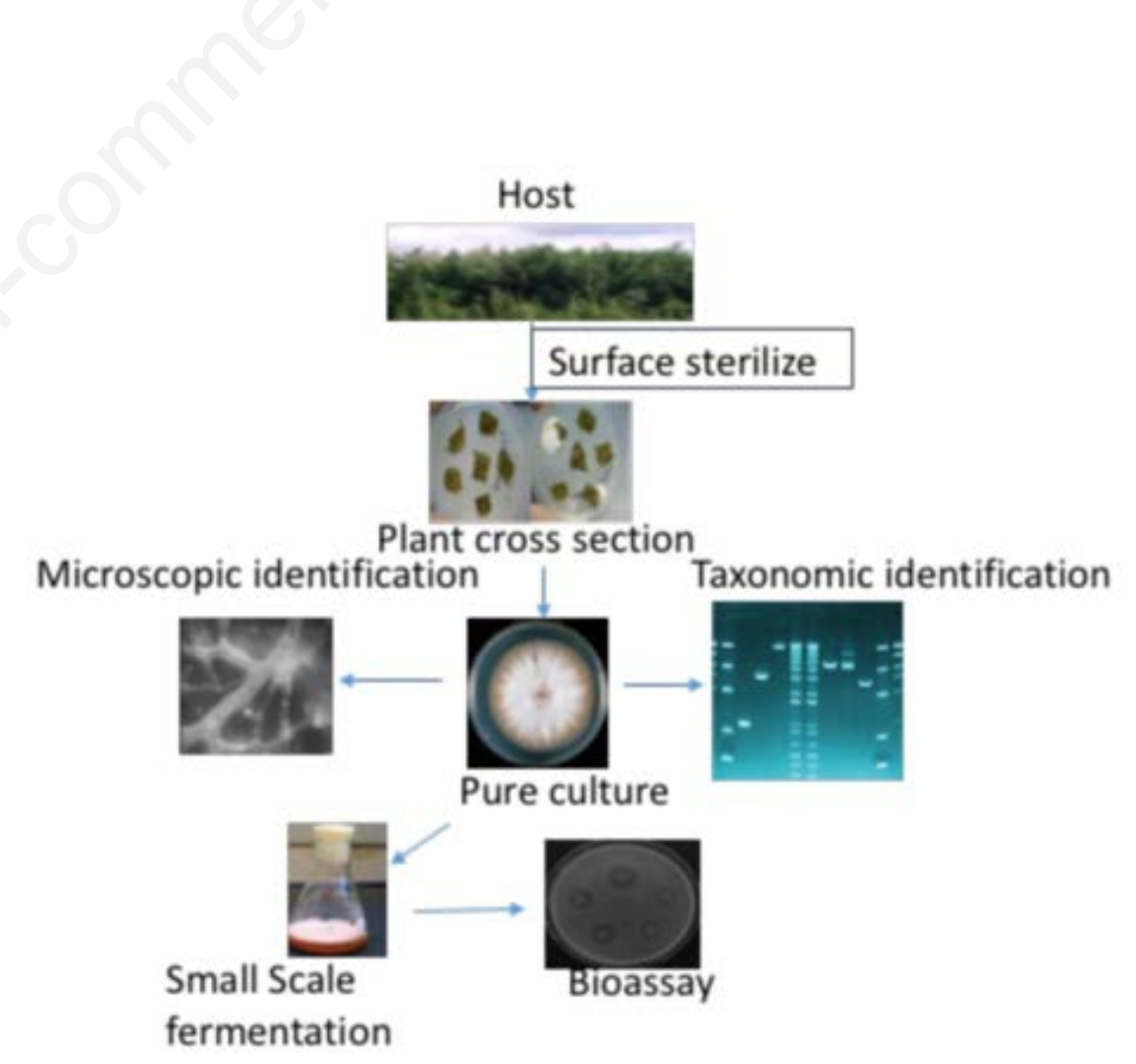

Figure 1. The interaction between endophytes and plant host. 
free radical scavenging ability of phenols is attributed to the occurrence of hydroxyl groups. Phenols and alkaloids were the major phytochemical constituents of endophytes and were reported by Huang et al. Cephalosporium sp., an endophytic fungus isolated from the root of Trachelospermum jasminoides (Apocynaceae) produce a phenolic compound (graphislactone A) with strong free radical scavenging and antioxidant activity. ${ }^{26}$ Cui et al., in 2015 isolated 315 endophytes from Rhodiola crenulata, $R$. angusta, and $R$. sachalinensis and reported that antioxidant assay by showing that the DPPH radical-scavenging rates of 114 isolates $(63.33 \%)$ were $>50 \%$, and those of five isolates (Rct45, Rct63, Rct64, Rac76, and Rsc57) were $>90 \% .{ }^{27}$ The culture filtrate of Pestalotiopsis microspora, which was isolated from combretaceaous plant Terminalia morobensis, showed promising antioxidant activity. ${ }^{28}$

\section{Antimicrobial activities of endo- phytes}

Many endophytic fungi have the ability to produce antimicrobial substances. An increasing number of compounds with antibacterial activity are being isolated from endophytic fungi, including fumitremorgins B isolated from Phomopsis sp., and periconicins A and B from Periconia sp. ${ }^{29}$ Research has shown that the compound ophiopogon japonicus pill has displayed effective inhibition of Staphylococcus aureus and an extract from O. japonicus showed strong inhibition of malic mildew. ${ }^{30}$ $8.3 \%$ of the strains isolated from Dracaena cambodiana and Aquilaria sinensis showed antimicrobial activity, ${ }^{31}$ whereas $27.6 \%$ of strains isolated from Camptotheca acuminata displayed antimicrobial activity against some pathogens. $^{32}$ Antimicrobial activity of endophytic fungi from Lippiasidoides Cham. showed antimicrobial activity in an assay on a solid medium. ${ }^{33}$ The steroid ergosta-7,9(14),22-triene$3 \beta$-ol, produced by the endophytic fungus Nigrospora sphaerica isolated from leaves of Vinca rosea, showed antifungal activity against Cryptococcus neoformans with an IC50 value of $14.81 \mu \mathrm{g} / \mathrm{mL} .{ }^{34}$ Maryam et al. reported Sixteen out of 23 bacterial isolates $(69 \%)$ exhibited antimicrobial activity against the selected pathogenic bacteria, such as Bacillus cereus, Staphylococcus aureus, Bacillus subtilis, Klebsiella pneumoniae, Citrobacter freundii, Proteus mirabilis, Shigella flexneri and Escherichia coli. ${ }^{35}$ Rodrigues et al., in 2000 have reported that Guignardia sp. from Spondias mombin inhibited E. coli, $S$. aureus,
Saccharomyces cerevisiae, Geotrichum $s p$. and Penicillium canadensis. ${ }^{36}$ Antimicrobial activity was exhibited by some endophytic fungal isolates from Cinnamomum. burmani, C. camphora, and C. insularimontanum and C. zeylanicum. ${ }^{37}$ Beauvericin, isolated from Fusarium oxysporum, which is associated with cinnamomum kanihirai, was reported toxic against methicillin-resistant $S$. aureus and B. subtilis. $^{38}$ The endophyte of Magnolia $x$ soulangeana, Fusarium dimerum, was the source of enniatins which exhibited effectiveness against Bacillus subtilis, Candida albicans, Trychosporom cutaneum and Cryptococcus neoformans. ${ }^{39}$

\section{Anticancer activities of endo- phytes}

There is some evidence that bioactive compounds produced by endophytes could be substitute approaches for innovation of new drugs, as various natural products of plants, microorganisms, and marine sources were identified as anticancer agents. Firakova et al., in 2007 reported diterpenoid that taxol also known as paclitaxel as a novel drug since it's due to the mode of action compared to other anticancer agents. ${ }^{40}$ Taxol is beneficial for the cure of advanced breast cancer, lungs cancer, and refractory ovarian cancer. Taxol was from the bark of tree belonging to taxus family (Taxus brevifolia), its main general resource. Another significant anticancer compound is the alkaloid Camptothecin $\left(\mathrm{C}_{20} \mathrm{H}_{16} \mathrm{~N}_{2} \mathrm{O}_{4}\right)$, a powerful antineoplastic agent which was firstly isolated from the wood of Camptotheca acuminate Decaisne (Nyssaceae) in China. ${ }^{41}$ Ergoflavin $\left(\mathrm{C}_{30} \mathrm{H}_{26} \mathrm{O}_{14}\right)$, a dimeric xanthene linked in position 2, belongs to the compound class called ergochromes and was described as a new anticancer agent isolated from an endophytic fungus rising on the Mimuscop elengi (Sapotaceae) plant leaves ${ }^{42} \mathrm{Wu}$ et al., in 2015 also reported leaf endophytes from Morinda citrifolia inhibited the growth of human carcinoma cell lines of lungs, prostate, and breast. ${ }^{43}$ Bioactive compound sclerotiorin has been isolated from an endophytic fungus Cephalotheca faveolata. ${ }^{44}$ Sclerotiorin was found to be potent antiproliferative against different cancer cells. An endophytic fungus Colletotrichum gloeosporioides (strain JGC-9) was isolated from Justicia gendarussa, a medicinal plant and screened for taxol production. ${ }^{45}$ Podophyllum hexandrum, which produced podophyllotoxin, podophyllotoxin glycoside and demethoxypodophyllotoxin. The isolated metabolites exhibited cytotoxicity in U-87 cell line. ${ }^{46}$ The production of podophyllotoxin from endophytic Alternaria tenuissima isolated from Sinopodophyllum emodi (Wall) was also reported by Liang et al. ${ }^{47}$

\section{Conclusions}

Endophytic fungi comprise a diverse group of species existing in various ecosystems and are capable of synthesizing bioactive compounds, which have proven to be useful for novel drug discovery. This group, which causes no apparent effects on plant performance but lives on the metabolites produced by the host, is presumably the most dominant functional group among endophytes by quantity. Since many microorganisms have developed resistance against the current drugs so it is necessary to search for new drugs. Much more work is essential to understand endophytes physiology, biochemical pathways, defensive role, secondary metabolite production, motivation and encouragement of researcher from life sciences to contribute research related to endophytes. It is vital importance to review and highlight the previous successes, on-going research and latest developments in research associated with endophytic microorganisms to draw the attention of the research community toward this emerging field and possible exploitation of the available sources for their therapeutic uses in various fields, such as the medical, pharmaceutical, food and cosmetics.

\section{References}

1. Carroll GC. Fungal endophytes in stems and leaves: from latent pathogen to mutualistic symbiont. Ecol 1988;69:2-9.

2. Parker MA. Mutualism in metapopulations of legumes and Rhizobia. Am Natural 1999;153:S48-60.

3. Dudeja SS, Giri R, Saini R, et al. Interaction of endophytic microbes with legumes. J Basic Microb 2012;52:248-60.

4. Stone JK, Polishook JD, White JF. Endophytic fungi. In: G.M. Mueller, G.F. Bills and M.S. Foster (Eds.), Biodiversity of fungi: inventory and monitoring methods. New York: Elsevier Academic Press; 2004. pp. 241-270.

5. Cheplick GP, Clay K. Acquired chemical defenses of grasses: the role of fungal endophytes. Oikos 1988;52:309-18.

6. Saikkonen K, Ion D, Gyllenberg M. The persistence of vertically transmitted fungi in grass metapopulations. Proc R Soc B Biol Sci 2002;269:397-1403. 
7. Saikkonen K, Faeth SH, Helander M, Sullivan TJ. Fungal endophytes: a continuum of interactions with host plants. Annu Rev Ecol Evol Syst 1998;29:319-43.

8. Faeth SH, Sullivan TJ. Mutualistic, asexual endophytes in a native grass are usually parasitic. Am Nat 2003;161: 310-25.

9. Rodriguez RJ, White JF, Arnold AE, Redman RS. Fungal endophytes: diversity and functional roles. New Phytol 2009; 182:314-30.

10. Wani ZA, Ashraf N, Mohiuddin T, RiyazUl-Hassan S. Plant-endophyte symbiosis, an ecological perspective. Appl Microbiol Biotechnol 2015;99: 2955-65.

11. Arnold AE, Lutzoni F. Diversity and host range of foliar fungal endophytes: are tropical leaves biodiversity hotspots? Ecology 2007;88:541-9.

12. Vasiliauskas R, Menkis A, Finlay RD, Stenlid J. Wood $\square$ decay fungi in fine living roots of conifer seedlings. N Phytol 2007;174:441-6.

13. Selosse MA, Vohník M, Chauvet E. Out of the rivers: are some aquatic hyphomycetes plant endophytes? N Phytol 2008;178:3-7.

14. Gamboa MA, Laureano S, Bayman P. Measuring diversity of endophytic fungi in leaf fragments: Does size matter? Mycopathologia 2003;156:41-5.

15. Lucero ME, Unc A, Cooke $P$, et al. Endophyte microbiome diversity in micropropagated Atriplex canescens and Atriplex torreyi var. griffithsii. PLoS One 2011 [Epub ahead of print].

16. Rai R, Dash PK, Prasanna BM, et al. Endophytic bacterial flora in the stem tissue of a tropical maize (Zea mays L.) genotype: isolation, identification and enumeration. World J Microbiol Biotechnol 2007;23:853-8.

17. Hata K, Sone K. Isolation of endophytes from leaves of Neolitsea sericea in broadleaf and conifer stands. Mycoscience 2008;49:229-32.

18. Youngbae S, Kim S, Park CW. A phylogenetic study of polygonum sect. tovara (polygonaceae) based on ITS sequences of nuclear ribosomal DNA. Plant Biol 1997;40:47-52.

19. Azevedo JL, Araujo WL. Genetically modified crops: environmental and human health concerns. Mutat Res 2003;544:22333.

20. Rosenblueth M, Martínez-Romero E. Bacterial endophytes and their interactions with hosts. Am Phytopathol Soc 2006;827-37.

21. Bing LA, Lewis LC. Suppression of Ostrinia nubilalis (Hübner) (Lepidoptera: Pyralidae) by endophytic Beauveria bassiana (Balsamo) Vuillemin. Environ Entomol 1991;20:1207-11.

22. Noel O, Nicholas H. Endophytes - the chemical synthesizers inside plants. Sci Progr 2004;87.

23. Tan RX, Zou WX. Endophytes: a rich source of functional metabolites. Nat Prod Rep 2001;18:448-59.

24. Afra K, Ietidal M, Justinne T, et al. A pilot study of the antioxidant potential of endophytic fungi from some Sudanese medicinal plants. Asian Pac J Trop Dis 2015;8:701-4.

25. Yadav M, Yadav A, Yadav JP. In vitro antioxidant activity and total phenolic content of endophytic fungi isolated from Eugenia jambolana Lam. Asian Pac J Trop Med 2014;7S1:S256-61.

26. Song YC, Huang WY, Sun C, et al. Characterization of graphislactone $\mathrm{A}$ as the antioxidant and free radical scavenging substance from the culture of Cephalosporium sp. IFB-E001, an endophytic fungus in Trachelospermum jasminoides. Biol Pharm Bull 2005;28: 506-9.

27. Cui JL, Guo TT, Ren ZX, et al. Diversity and Antioxidant Activity of Culturable Endophytic Fungi from Alpine Plants of Rhodiola crenulata, R. angusta, and R. sachalinensis. PLoS One 2015;10:3.

28. Harper JKEJ, Ford GA, Strobel A, et al. Pestacin: A 1,3-dihydro isobenzofuran from Pestalotiopsis microspora possessing antioxidant and antimycotic activities. Tetrahedron 2003;59: 2471-6.

29. Ni ZW, Li GH, Zhao PJ, Shen YM. Antimicrobial components of the endophytic fungal strain chaetomium globosum Ly50' from maytenus hookeri. Nat Prod Rev 2008;20:33-6 [in Chinese].

30. Rao Y, Chen C, Zhao B. Study on the eliminating phlegm effect and bacteriostatic action of compound Maidong pills. Chin Hosp Pharm J 2008;28:615-7.

31. Gong LJ, Guo SH. Endophytic fungi from Dracaena cambodiana and Aquilaria sinensis and their antimicrobial activity. Afr J Biotechnol 2009;8: 731-6.

32. Lin X, Lu C, Huang Y, et al. Endophytic fungi from a pharmaceutical plant, Camptotheca acuminata: isolation, identification and bioactivity. World J Microbiol 2007;23:1037-40.

33. Siqueira VM, Conti R, Araújo JM, SouzaMotta CM. Endophytic fungi from the medicinal plant Lippia sidoides Cham. and their antimicrobial activity. Symbiosis 2011;53:89-95.

34. Metwaly AM, Kadry HA, El-Hela AA, et al. Nigrosphaerin A a new isochromene derivative from the endophytic fungus Nigrospora sphaerica. Phytochem Lett 2014;7:1-5.

35. Maryam B, Mansour A, Abdolrazag H, et al. Antimicrobial activity of endophytic bacterial populations isolated from medical plants of Iran. Iran J Microbiol
2017;9:11-8.

36. Rodrigues KF, Hesse M, Werner C. Antimicrobial activities of secondary metabolites produced by endophytic fungi from Spondias mombin. J Basic Microbiol 2000;40:261-7.

37. Praptiwi, Ilyas M, Fathoni A, et al. Antibacterial screening of the culture of endophytic fungal extracts isolated from cinnamon stick. Teknol Indones 2015;38:33-41.

38. Wang QX, Li SF, Zhao F, et al. Chemical constituents from endophytic fungus Fusarium oxysporum. Fitoterapia 2011;82:777-81.

39. Firakova S, Sturdikoca M, Liptaj T, Bezakova L, Proksa B. Enniatins produced by Fusarium dimerum, an endophytic fungal strain. Pharmazie 2008; 63:539-41.

40. Firakova S, Sturdikoca M, M'ǔckov'a. Bioactive secondary metabolites produced by microorganisms associated with plants. Biologia 2007;62:251-7.

41. Cremasco MA, Hritzko BJ, Linda WNH. Experimental purification of paclitaxel from a complex mixture of taxanes using a simulated moving bed. Braz J Chem Eng 2009;26:207-18.

42. Deshmukh S, Mishra PD, KulkarniAlmeida S, et al. Anti-inflammatory and anticancer activity of Ergoflavin isolated from an endophytic fungus. Chem Biodivers 2009;6:784-9.

43. Wu Y, Girmay S, da Silva VM, et al. The Role of Endophytic Fungi in the Anticancer Activity of Morinda citrifolia Linn. (Noni). Evid Based Complement Alternat Med 2015;2015: 393960.

44. Periyasamy G, Shilpa AV, Amit K, et al. Anticancer activity of sclerotiorin, isolated from an endophytic fungus Cephalotheca faveolata. Indian J Exper Biol 2012;50: 464-8.

45. Gangadevi V, Muthumary J. Isolation of Colletotrichum gloeosporioides, a novel endophytic taxol producing fungus from the leaves of a medicinal plant, Justicia gendarussa. Mycol Balcan 2008;5:1-4.

46. Puri S, Nazir Asiya N, Chawla R, et al. The endophytic fungus Trametes hirsuta as a novel alternative source of podophyllotoxin and related aryl tetralin lignans. J Biotechnol 2006;122:494-510.

47. Liang Z, Zhang JX, Zhang X, et al. Endophytic Fungus from Sinopodophyllum emodi (Wall.) Ying that Produces Podophyllotoxin. J Chromatogr Sci 2016;54:175-8. 